\title{
Review
}

\section{Technology for Measuring and Monitoring Treatment Compliance Remotely}

\author{
Richard H. Christie, Anzar Abbas and Vidya Koesmahargyo* \\ AiCure, New York, NY, USA
}

Accepted 27 May 2021

Pre-press 17 June 2021

\begin{abstract}
Medication non-adherence during clinical trials is an ongoing challenge that can result in insufficient safety and efficacy data. For patients with Parkinson's disease and other neurological disorders, symptomatology such as forgetfulness compounds traditional obstacles to adherence. Today, sponsors and clinical study sites can call upon various technology tools that improve adherence by monitoring and confirming dosage in near real-time. These tools have the potential to improve the quality of data gleaned from these studies.
\end{abstract}

Keywords: Digital phenotyping, Parkinson's disease, remote measurement, treatment adherence

\section{INTRODUCTION}

Treatment compliance, or adherence, is a critical issue in clinical trials. Non-adherence by patients with chronic conditions is linked to higher healthcare costs, increased patient and societal burden and reduced quality of life, in general, thus it has become a focus for trial sponsors to understand how to engage patients and optimize medication adherence.

For patients with Parkinson's disease, suboptimal adherence is common and varies between 10 and $67 \%$ [1]. Non-adherence from patients happens for a wide variety of reasons. These include, but are not limited to:

- Issues or complications due to the illness itself

- Issues or complications due to other morbidities

- Non-medical issues, such as career and family obligations or transportation challenges

With neurological disorders such as Parkinson's disease, mood components also present that can be

${ }^{*}$ Correspondence to: Vidya Koesmahargyo, 100 S. Bedford Rd., Suite 340, Mt. Kisco, NY 10549, USA. Tel.: +1 646301 5037; E-mail: vidya.koesmahargyo@aicure.com. obstacles to treatment adherence [2]. Any type of patient may have trouble remembering facets of their treatment regimen or keeping a consistent routine. These factors can be particularly challenging for patients dealing with Parkinson's and similar neurological diseases.

\section{UNDERSTANDING WHY TREATMENT NON-ADHERENCE OCCURS}

Even well-organized and reliable patients will miss an occasional dose, or even stop taking their medication altogether. The reasons for this range from lifestyle impacts such as work and family to specific health reasons. For example, a patient may begin to feel sicker and attribute the change to the medication, causing them to take a day or two off or even stop medicating entirely. They may experience a turn for the better, feel healthy and thus stop dosing. While these things happen during trials and are to be expected, they become challenging for clinical trial sites who may not have day-to-day insight into the patient's behavior and adherence. If a week or a month goes by without the site knowing about these 
kinds of changes, it can negatively impact study data, and potentially study outcomes

\section{BENEFITS OF REMOTE MONITORING AND ENGAGEMENT}

The ability to monitor medication adherence remotely, capturing data from patients at home, presents numerous benefits for clinical study teams. In older study models, data collection relied heavily on in-person clinical visits, complemented by patient diaries. Trusting patients with keeping accurate and timely data on medication adherence is problematic regardless of the therapeutic area being studied but presents acute challenges in patients for whom unreliable memory is a primary symptom. Solutions such as self-reporting (as in diaries or in clinical visits) and pill counts have proven weak indicators of adherence in patients with Parkinson's disease [3]. Further, symptoms of Parkinson's disease can fluctuate and therefore cannot always be recorded reliably during clinical evaluations [4]. Remote monitoring and engagement technologies represent a way for study teams to gain insight into those time gaps between clinical visits. Motor symptoms such as tremor can now be remotely monitored in real-time and shared with the clinical team. Caregivers would then have a more complete history of symptomatology for each patient and would be less likely to miss important information should the patient show mild or no symptoms during a clinic visit. Data gleaned from remote monitoring technology also serves to help clinicians identify potential problems more quickly.

\section{TECHNOLOGY SOLUTIONS FOR TREATMENT NON-ADHERENCE}

Technologies exist today to engage patients with added support and reduced monitoring burden for clinical trial sites. These technologies include approaches such as "Smart" pill packs, wearable devices which transmit a wide range of patient data to sites, and remote monitoring and engagement applications that can be used by patients through smartphones or similar devices, either those they already own or devices provided by trial sponsors or facilitators.

\section{“Smart” pill packs}

This technology utilizes packaging outfitted with electronics that wirelessly track pill usage and transmit that data to an application on a computer or smart device, where it is then forwarded to a database utilized by the study team [5]. When pills are removed from the pack, data such as type of pill, position in the pill pack, and data and time of removal are recorded. If pills are not removed according to the assigned treatment plan, reminders can be generated to alert the patient. Similarly, study teams can be alerted if not enough, or too many doses are removed within a given time window, allowing them to reach out to patients when necessary. While a useful step forward, these pill packs are not able to track if the pill was actually taken by the patient after its removal from the pack.

\section{Wearables}

Wearable devices are currently being researched for their utility in improving adherence. For example, one group of researchers is seeking to complement the abilities of "smart" pill packs with a way to confirm ingestion of the medication via a necklace that would detect throat movement [6]. For Parkinson's disease, specifically, developers of the Apple Watch are building capabilities to detect changes in tremors associated with the disease [7]. While this may not be able to confirm dosing, it may be useful in the future in pinpointing improvements to symptomatology resulting from therapy.

\section{Smart device applications}

Applications are now being used in clinical trials with proven utility and accuracy to confirm dosing adherence remotely. Such applications utilize the patients' own smart devices or devices provided by sponsors or facilitators. Through the application, patients are reminded to take their medication and given guidance on how to properly take it. Further, the video recording capabilities of the smart device are paired with proprietary artificial intelligence technologies that record each dose with the ability to confirm the correct pill is being taken and that it is successfully ingested by the patient [8]. Patients are walked through a series of dosing steps, each of which is confirmed by on-phone computer vision models that identify successful completion of the step. By confirming the correct pill, its placement on the tongue, and an open-mouth check following ingestion, the technology can remotely perform actions similar to an in-person Directly Observed Therapy assessment. 
Patient 1: 73\%

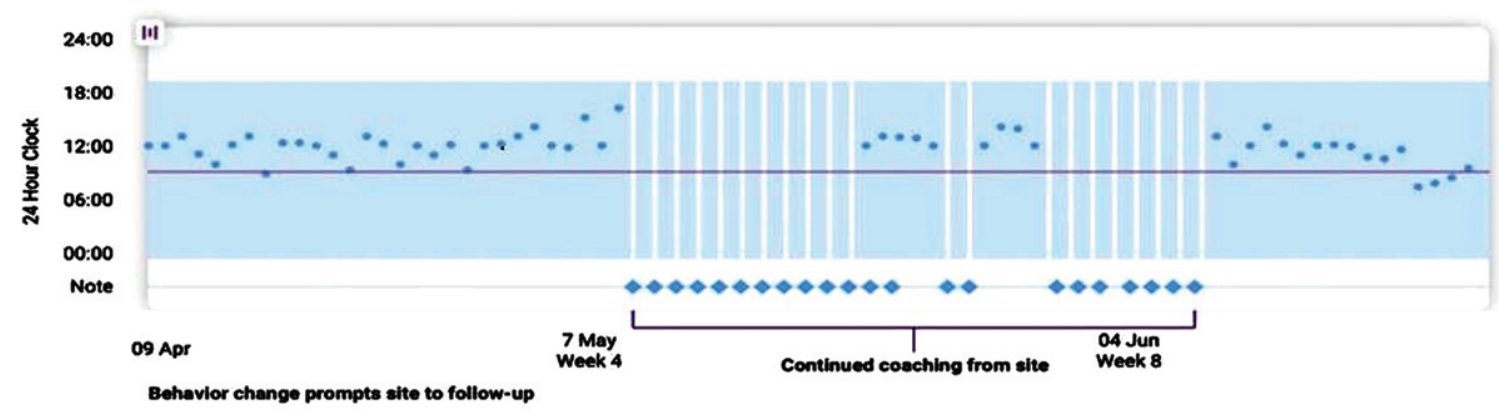

\section{Patient 2: 83\%}

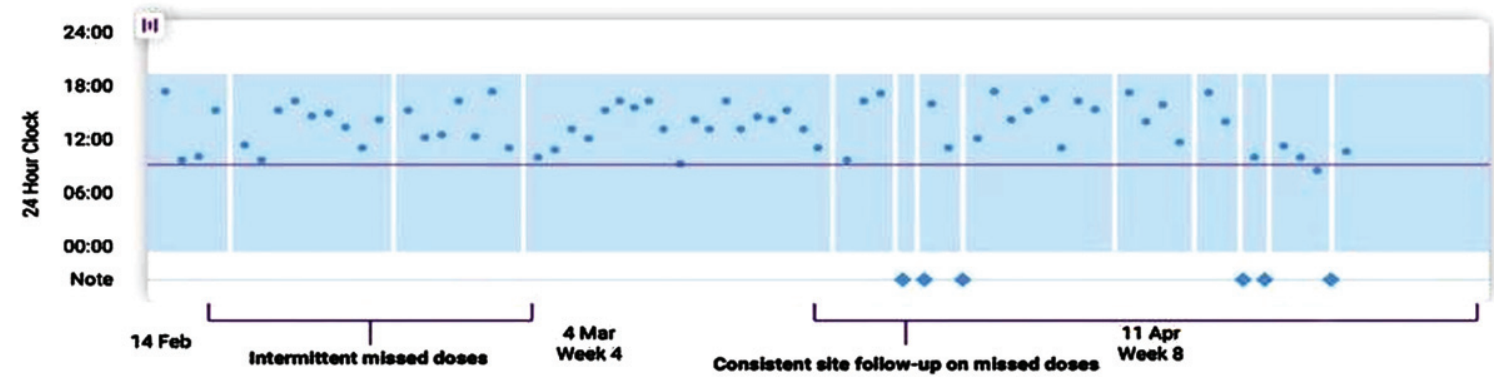

Patient 3: 100\%

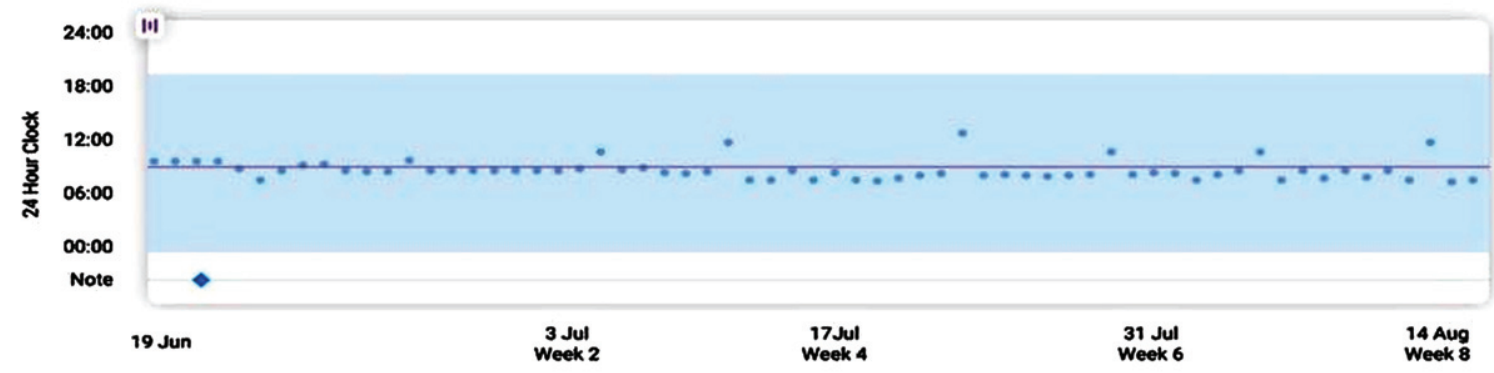

Fig. 1. An example of a visual dashboard into patient adherence activity recorded through a remote monitoring and engagement application (AiCure). In Patient 1, we see that behavior changes detected through the remote technology prompted the study site to lend additional support. Similarly, intermittent missed doses by Patient 2 resulted in continued study team follow-up.

These applications also facilitate quick and easy access to site support, allowing patients and sites to interact in a way that alleviates questions and stress for patients. This can alleviate site burden, as communications via the app can be less intrusive than following up by the phone or requiring additional clinical visits. Sites and sponsors receive real-time data on adherence and can quickly identify any changes to the patient's dosing behavior. This allows sites to engage with patients to understand and work to solve adherence problems quickly. (See Fig. 1 below). Importantly, new evidence demonstrates that direct remote measurements of adherence can be used directly to make dynamic predictions about adherence behavior in the future [9].

\section{Implications of remote monitoring of adherence}

As with the introduction of any new technology, many questions are raised about the possible impact of the technology to existing processes and protocols. One open question is to what extent the use of the technologies outlined here will indeed drive increased adherence in Parkinson's disease patients. One recent experience with technology for remote support of aerobic exercise in Parkinson's disease, 
for example, suggests that in-app gamification or other forms of built-in incentivization may augment patients' engagement with the app, supporting the resulting desired behavior [10]. An additional question is to what extent these technologies are able to be 'tricked' into showing compliance, and whether there may be a sense of 'false security' from the use of these remote monitoring approaches. Indeed, a recent article suggested that some percentage of doses were 'intentionally non-adherent' where patients sought to circumvent the phone-based technology for dosing support [11]. Additional data is needed about the prevalence of this behavior in specific patient populations and clinical scenarios to understand the true impact of these technologies on trials and in realworld deployments.

While overall, the use of adherence technology is a benefit to clinical trials in that it ensures data quality, several issues are raised that will continue to require study. One is the possible impact of dosing monitoring on dosing itself. The well-documented 'Hawthorne Effect' speaks to this possibility, that the very act of observing dosing increases compliance rates among patients being monitored. On the one hand, this can result in clinical trials whose execution more closely hews to the design of the trial protocol. Conversely, this may raise questions related to how applicable the dosing will be to real-world use post approval. The increased use of dosing monitoring technologies in real-world conditions will ultimately provide the data required to understand and appreciate the impact of dosing monitoring on real-world adherence.

\section{CONCLUSION}

While medication non-adherence will always be a challenge for any clinical trial, technology can be utilized to help improve treatment compliance. For patients dealing with Parkinson's disease, technologies that provide easy to follow dosing instructions and reminders, along with the ability to accurately track successful medication ingestion have the potential to increase adherence, providing better study data for sponsors.

\section{Take home points}

- Medication adherence is a common challenge for patients with Parkinson's disease with suboptimal adherence ranging widely (between 10\%-67\%).
- Reasons for non-adherence include symptomatology of Parkinson's disease itself, the presence of other morbidities and lifestyle challenges such as work and family.

- Remote monitoring and engagement technologies are being employed to help improve adherence. Some of these technologies are already in use, such as "smart" pill packs and applications that monitor and confirm dosing. More research is being done to study the impact of additional technologies such as wearable devices on improving medication adherence.

- Technologies that can assist patients with medication adherence and accurately confirm successful dosing provide valuable data to study sites and sponsors and allow sites to engage patients when doses are missed.

\section{CONFLICT OF INTEREST}

RHC, AA, and VK are employees of AiCure and eligible for equity-based compensation in that regard.

\section{REFERENCES}

[1] Malek N, Grosset DG (2015) Medication adherence in patients with Parkinson's disease. CNS Drugs 29, 47-53.

[2] Daley DJ, Myint PK, Gray RJ, Deane KHO (2012) Systematic review on factors associated with medication non-adherence in Parkinson's disease. Parkinsonism Relat Disord 18, 1053-1061.

[3] Grosset KA, Bone I, Reid JL, Grosset D (2006) Measuring therapy adherence in Parkinson's disease: A comparison of methods. J Neurol Neurosurg Psychiatry 77, 249-251.

[4] Heldman DA, Harris DA, Felong T, Andrzejewski KL, Dorsey ER, Giuffrida JP, Goldberg B, Burack MA (2017) Telehealth management of Parkinson's disease using wearable sensors: An exploratory study. Digit Biomark 1, 43-51.

[5] Poor A (2018) Smart pill pack to improve adherence. HealthTech Insider, https://healthtechinsider.com/ 2018/08/15/smart-pill-pack-to-improve-adherence/

[6] Kalantarian H, Motamed B, Alshurafa N, Sarrafzadeh M (2016) A wearable sensor system for medication adherence prediction. Artif Intell Med 69, 43-52.

[7] Monitoring Movement Disorders | Apple Developer Documentation.

[8] Bain EE, Shafner L, Walling DP, Othman AA, ChuangStein C, Hinkle J, Hanina A (2017) Use of a novel artificial intelligence platform on mobile devices to assess dosing compliance in a phase 2 clinical trial in subjects with schizophrenia. JMIR MHealth UHealth 5, e18.

[9] Koesmahargyo V, Abbas A, Zhang L, Guan L, Feng S, Yadav V, Galatzer-Levy IR (2020) Accuracy of machine learning-based prediction of medication adherence in clinical research. Psychiatry Res 294, 113558.

[10] van der Kolk NM, de Vries NM, Kessels RPC, Joosten H, Zwinderman AH, Post B, Bloem BR (2019) Effectiveness 
of home-based and remotely supervised aerobic exercise in Parkinson's disease: A double-blind, randomised controlled trial. Lancet Neurol 18, 998-1008.
[11] Getz K, Smith Z, Shafner L, Hanina A (2020) Assessing the scope and predictors of intentional dose non-adherence in clinical trials. Ther Innov Regul Sci 54, 1330-1338. 